\title{
CLIN I CAL VIDEO
}

\section{Catastrophic Cough in a Young Infant}

A 45-day-old girl child presented with cough for 10 days, with increasing frequency and duration of cough bouts over the past two days. There was no fever or respiratory distress. Child had characteristic 'whooping cough'prolonged cough bouts ending in inspiratory whoop (Web Video 1 and Fig. 1). Complete blood count showed total leukocyte count of 26800 cells $/ \mathrm{mm}^{3}$ with $75 \%$ lymphocytes. Chest $X$-ray was normal. Child was treated with oral azithromycin for 5 days, suspecting pertussis. On day 2 of hospitalization, child had prolonged cough bouts associated with hypoxia and apneic spells requiring intubation. Nasopharyngeal swab for Bordetella pertussis PCR was reported to be positive. Child was extubated after 4 days of mechanical ventilation as the frequency of cough bouts on ventilator reduced. Child needed oxygen support during cough bouts even after extubation, and was gradually weaned off oxygen over 14 days. At discharge, child had cough bouts with reduced severity and no hypoxia. Further questioning revealed that child was scheduled for her first dose of DTP vaccine next week and mother had received TT vaccine, not Tdap, during her antenatal visits. On follow-up, child was thriving well, had occasional cough episodes, and had been immunized with 3 doses of DTP vaccine.

Cough in children may sound benign, but sometimes it may be harbinger of serious underlying diseases like pneumonia, bronchiolitis or Pertussis. Differential diagnosis of child with prolonged acute cough include

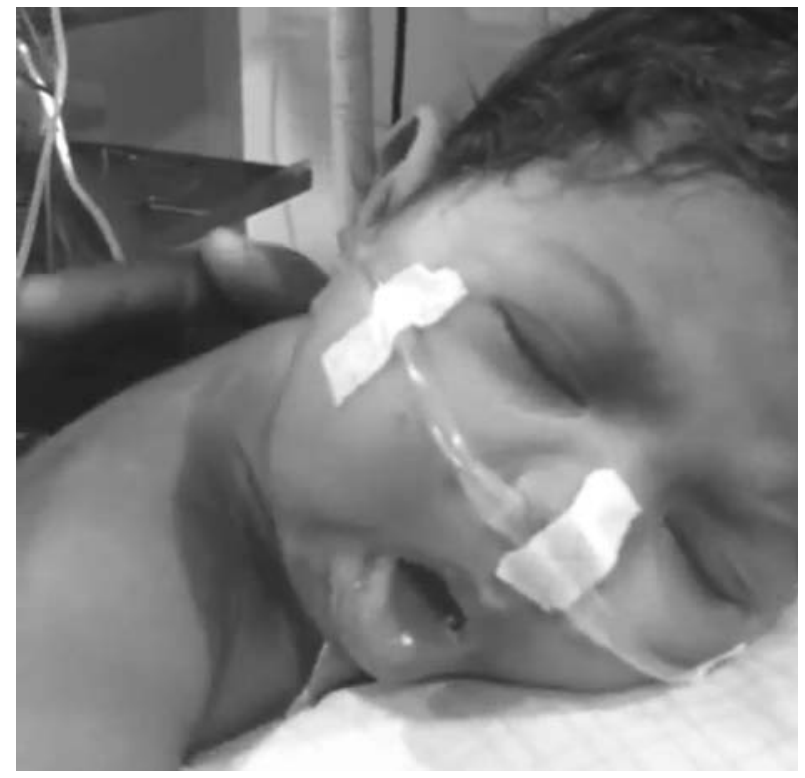

FIG. 1 Infant with whooping cough .

Postviral cough, Pertussis, Persistent bacterial bronchitis, Bronchiolitis, Pneumonia or Retained foreign body. Pointers useful in differentiating pertussis from these conditions include characteristic inspiratory whooping sound of cough, cough bouts escalating at 7-10 days, apneic spells or cyanosis in infants, and presence of leukocytosis with $>50 \%$ lymphocytes.

$$
\begin{array}{r}
\text { B BANUPRIYA }{ }^{1} \text { AND AJAGADEESH }{ }^{2} \\
\text { Department of Paediatrics, } \\
\text { Mahatma Gandhi Medical College and Research Institute, } \\
\text { Sri Balaji Vidyapeeth University, Puducherry, India. } \\
{ }^{1} \text { banu.13@gmail.com }
\end{array}
$$

\section{Notice}

\section{Call for Submission of 'Clinical Videos'}

Under this section, Indian Pediatrics publishes videos depicting an intricate technique or an interesting clinical manifestation, which are difficult to describe clearly in text or by pictures. A video file submitted for consideration for publication should be of high resolution and should be edited by the author in final publishable format. MPEG or MP4 formats are acceptable. The maximum size of file should be 20 MB. The file should not have been published elsewhere, and will be a copyright of Indian Pediatrics, if published. For this section, there should be a write-up of up to 250 words discussing the condition and its differential diagnoses. The write-up should also be accompanied by a thumbnail image for publication in the print version and PDF. Submit videos as separate Supplementary files with your main manuscript. A maximum of three authors (not more than two from a single department) are permissible for this section. In case the video shows a patient, he/she should not be identifiable. In case the identification is unavoidable, or even otherwise, each video must be accompanied by written permission of parent/guardian, as applicable. Authors are responsible for obtaining participant consent-to-disclose forms for any videos of identifiable participants, and should edit out any names mentioned in the recording. The consent form should indicate its purpose (publication in the journal in print and online, with the understanding that it will have public access) and the signed consent of the parent/legal guardian. The copy of the consent form must be sent as supplementary file along with the write-up, and original form should be retained by the author. A sample consent form is available at our website www.indianpediatrics.net. 